\title{
Impurity Resonances and the Origin of the Pseudo-Gap
}

\author{
Brian Møller Andersen \\ Ørsted Laboratory, Niels Bohr Institute, Universitetsparken 5, DK-2100 Copenhagen Ø, Denmark
}

Received on 23 May, 2003.

\begin{abstract}
We study the structure of resonance states localized around nonmagnetic impurities in the $\mathrm{CuO}_{2}$ planes of the cuprate superconductors within a potential scattering formalism. In particular we show that strong quantum interference effects arise between several impurities. This interference can be utilized to distinguish the dwave superconducting state from the phase with d-density wave order. This is important if the origin of the pseudo-gap state in the underdoped regime of the High $\mathrm{T}_{c}$ superconductors is caused by preformed Cooper pairs or staggered orbital currents. Furthermore impurity interference can be utilized to reveal subdominant superconducting order parameters and to pose further constraints on the potential scattering scenario.
\end{abstract}

For conventional superconductors Yu and Shiba[1] first showed that as a result of the interaction between a magnetic impurity and the spin density of the conduction electrons, a bound state located around the magnetic impurity is formed inside the gap in the strong-scattering (unitary) limit. For anisotropic superconductors a number of authors generalized the Yu-Shiba approach to study the effects of single impurities[2].

Many important questions concerning the electronic structure of the High $\mathrm{T}_{c}$ materials still need to be answered. The study of single impurity effects provide a promising path to yield some answers. This is mainly due to the large experimental progress in low temperature scanning tunneling microscopy (STM). In particular, STM measurements have provided detailed local density of states (LDOS) images around single nonmagnetic[3, 4] (Zn) and magnetic[5] (Ni) impurities on the surface of the high temperature superconductor $\mathrm{Bi}_{2} \mathrm{Sr}_{2} \mathrm{CaCuO}_{4+\delta}$ (BSCCO). More recently the energy dependence of the Fourier transformed LDOS images was measured in the superconducting state of optimally doped $\mathrm{BSCCO}[6]$. The dispersive features were explained by elastic quasi-particle interference resulting from a single weak, nonmagnetic impurity[7]. This gives credence that a scattering potential picture can yield valuable predictions in the superconducting state of these materials. Furthermore, it was recently shown by Martin et al.[8] that both the energetics of the resonance state and its spatial dependence around a strong potential scatterer (e.g. Zn) can be accounted for by including the tunnelling (the filter) through excited states from the $\mathrm{CuO}_{2}$ planes to the top $\mathrm{BiO}$ layer probed by the STM tip. In order to obtain agreement with the measured LDOS one needs to attribute a large negative potential to the $\mathrm{Zn}$ site in agreement with the filled d shell of this atom; the potential will strongly repel holes, i.e. attract the electrons.
Experimentally there is also evidence from NMR measurements that magnetic moments are induced around nonmagnetic impurities[9]. In this paper we assume, however, that the large potential scattering off the impurity site itself is dominating the final LDOS.

Future experimental ability to control the position of the impurities on the surface of a superconductor and perform detailed STM measurements around multiple impurity configurations motivates theoretical studies of the interference between the impurity wavefunctions. For instance, this involves the physics of quantum mirages[10] and impurity effects in superconductors $[11,12,13]$. Below we compare the expected LDOS around one and two strong nonmagnetic impurities. Our main results are the following: 1) investigation of the LDOS around two impurities can pose strong constraints on the potential scattering model, 2) quantum interference can be utilized as an alternative method to detect small subdominant superconducting order parameters, and 3) quantum interference between two nonmagnetic impurities may easily distinguish between the d-wave superconducting (DSC) and d-density wave (DDW) states. The DDW state was recently proposed as a model for the pseudogap state of the cuprates[14]. Any difference in the impurity modified LDOS between the DSC and DDW states may reveal the hidden DDW order and distinguish between the scenario of preformed pairs versus staggered orbital currents as the origin for the pseudo-gap state[15]. Recently, there has been several other proposals to probe the DDW order in the cuprates[14, 16].

In attempting to make better contact to the experiments we include the tunneling through excited states from the $\mathrm{CuO}_{2}$ planes to the $\mathrm{BiO}$ layer probed by the STM tip[8]. This effect modifies the LDOS, $\rho(\mathbf{r}, \omega)=$ $\sum_{n}\left|\psi_{n}(\mathbf{r})\right|^{2} \delta\left(\omega-\epsilon_{n}\right)$, by including the four nearest $\mathrm{Cu}$ 
neighbors in the underlying $\mathrm{CuO}_{2}$ plane, $\psi_{n}(\mathbf{r}) \longrightarrow \psi_{n}(\mathbf{r}+$ $\left.\mathbf{e}_{x}\right)+\psi_{n}\left(\mathbf{r}-\mathbf{e}_{x}\right)-\psi_{n}\left(\mathbf{r}+\mathbf{e}_{y}\right)-\psi_{n}\left(\mathbf{r}-\mathbf{e}_{y}\right)$. Here $\mathbf{e}_{i}$ denote the unit vectors in the $\mathrm{CuO}_{2}$ layer.

The presence of scalar impurities is modelled by the following delta-function potentials

$$
\hat{H}^{i n t}=\sum_{\{i\} \sigma} U_{i} \hat{n}_{i \sigma},
$$

where $\hat{n}_{i \sigma}$ is the density operator on site $i$. Here $\{i\}$ denotes the set of lattice sites hosting the impurities and $U_{i}$ is the strength of the corresponding effective potential. In agreement with the above discussion the impurities are modelled with a potential, $U=-15 t$, corresponding to $-4.5 \mathrm{eV}$, which generates resonances at a few $\mathrm{meV}$ for a single nonmagnetic impurity[8]. The large scale of this potential renders the effects on the LDOS from corrections to other energy scales around the impurity site less important. For instance, we have checked that gap suppression near the impurity or slightly larger spatial extension of the impurity does not qualitatively affect the results reported below. In general these effects tend to push the resonances slightly further toward zero bias.

The full Greens function $\hat{G}(\mathbf{r}, \omega)$ can be obtained by solving the Gorkov-Dyson equation

$$
\hat{G}(\omega)=\hat{G}^{0}(\omega)\left(\hat{I}-\hat{H}^{i n t} \hat{G}^{0}(\omega)\right)^{-1}
$$

where $G^{0}$ is the Greens function for the clean system. The differential tunnelling conductance is proportional to the LDOS which in turn is determined from the imaginary part of the full Greens function. The size of the matrices in Eqn. (2) depends on the number of impurities and the dimension of the Nambu space. We have previously utilized this method to study the electronic structure around impurities and vortices that operate as pinning centers of surrounding stripes[13, 19].

The clean Greens function $\hat{G}^{0}\left(\mathbf{k}, i \omega_{n}\right)$ for the unperturbed d-wave superconductor is given by

$$
\hat{G}^{0}\left(\mathbf{k}, i \omega_{n}\right)=\left[i \omega_{n} \hat{\tau}_{0}-\xi(\mathbf{k}) \hat{\tau}_{3}-\Delta(\mathbf{k}) \hat{\tau}_{1}\right]^{-1},
$$

where $\hat{\tau}_{\nu}$ denotes the Pauli matrices in Nambu space, $\hat{\tau}_{0}$ being the $2 \times 2$ identity matrix, and $\omega_{n}$ is a Matsubara frequency. For a system with $\mathrm{d}_{x^{2}-y^{2}}$-wave pairing, $\Delta(\mathbf{k})=$ $\frac{\Delta_{0}}{2}\left(\cos \left(k_{x}\right)-\cos \left(k_{y}\right)\right)$. Below, $\Delta_{0}=50 \mathrm{meV}$ and the lattice constant is set to unity. The large value of the gap $\Delta_{0}$ corresponds roughly to the experimentally measured maximum gap in the underdoped regime of BSCCO. In agreement with photoemission we use a normal state dispersion $\xi(\mathbf{k})=\epsilon(\mathbf{k})-\mu$,

$$
\xi(\mathbf{k})=-2 t\left(\cos \left(k_{x}\right)+\cos \left(k_{y}\right)\right)-4 t^{\prime} \cos \left(k_{x}\right) \cos \left(k_{y}\right)-\mu
$$

with $t=300 \mathrm{meV}, t^{\prime}=-0.3 t$ and $\mu=-0.9 t$ which is relevant for BSCCO around $10 \%$ hole doping. In Eqn. (4) $\left(t^{\prime}\right) t$ refers to the (next)nearest neighbor hopping and $\mu$ is the chemical potential. Since it remains controversial which band applies to the DDW state[15] we also study the nested band, $t^{\prime}=0.0$ and $\mu=0.0$. In fact both cases may be relevant given the recent detection of strong spatial inhomogeneity in the density of states[18].

In the DSC state, the resonance condition $1=$ $U \operatorname{Re}\left[G^{0}\left(0, \pm i \omega_{n}\right)\right]$ for a single nonmagnetic impurity generates virtual bound states at positive and negative energies with respect to the Fermi level. However, the majority of the quasi-particle weight may reside on only one of these resonances[13]. It is evident from Fig. 1 that indeed only one resonance has weight which is contrary to the situation without the filter[15].

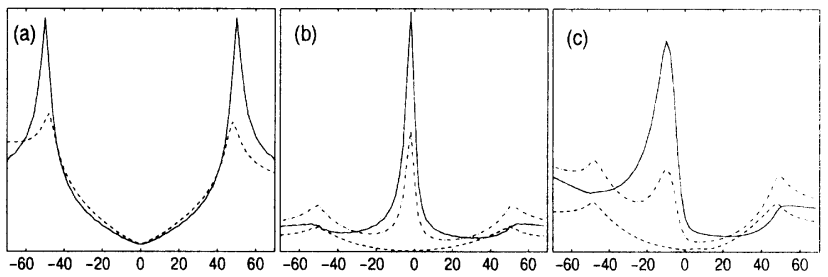

Figure 1. DOS (arb. units) vs energy (meV) around a single impurity in the DSC state. The impurity is positioned at $(0,0)$ and the graphs correspond to the DOS at $(0,0)$ (solid), $(1,0)$ (dashed), and $(1,1)$ (dash-dotted).

For interference between two nonmagnetic unitary impurities Morr et al.[11] found strong variations in the LDOS as the distance between the impurities $R$ is varied along one of the crystal axes. In the following we elaborate on the discussion of two nonmagnetic impurities in the DSC phase by a numerical study of the LDOS including the filtering effect. In Fig. 2 we show the density of states measured above one of the impurities fixed at the origin while the other is moved away along the nodal (a) or anti-nodal (b) direction. As expected for a $d_{x^{2}-y^{2}}$-wave superconductor, the length scale at which the single-impurity DOS recovers is much larger along the nodal directions. The single impurity DOS is obtained for $R$ well above 100 lattice constants. Thus only for impurity concentrations below $0.1 \%$ does the LDOS correspond to the expected result from a single strong nonmagnetic impurity. For weaker scatterers the decay length will be considerably reduced. For two impurities fixed at $( \pm 1,0)$ and the STM tip scanned from $(0,0)$ to $(8,0)$, Fig. 3 shows the expected LDOS. The number of apparent resonances, their energetic position and width strongly depend on the impurity configuration and the position of the STM tip. In particular, for certain tip positions the resonances completely disappear. 

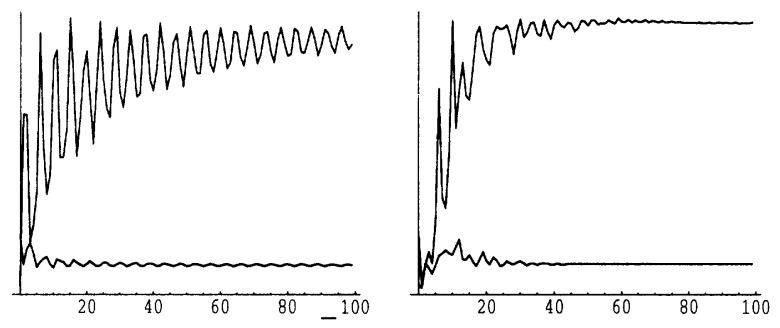

Figure 2. DOS at $(0,0)$ and at the single-impurity resonance energy $\pm 1.5 \mathrm{meV}$ as a function of distance between the two nonmagnetic impurities separated along the (left) nodal direction and (right) anti-nodal direction. The y-axis scale is identical for the two figures.
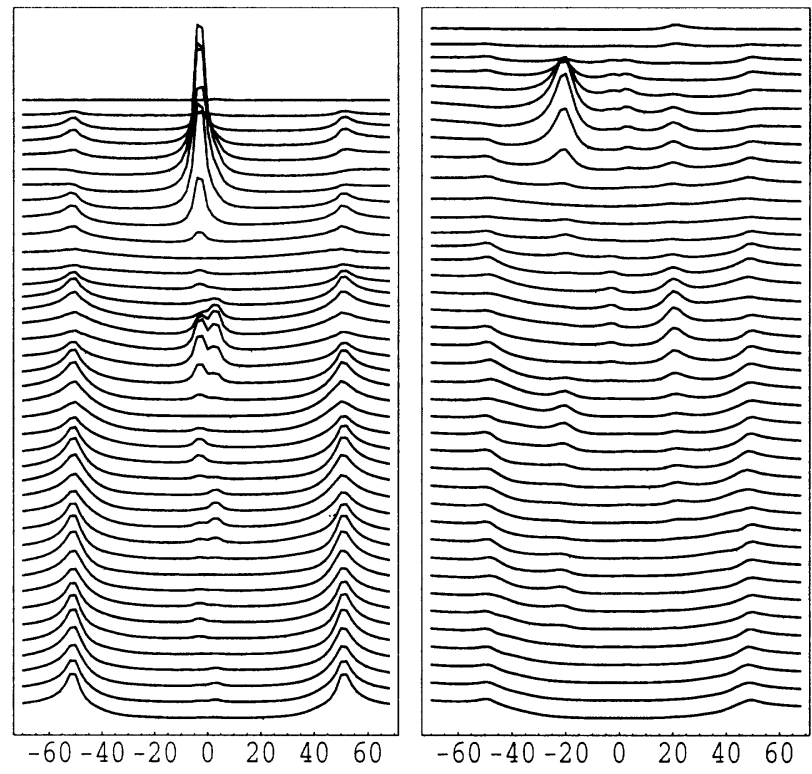

Figure 3. The impurities are fixed at $( \pm 1,0)$ while the STM tip is moved from $(0,0)$ (top) to $(8,0)$ (bottom) in steps of 0.2. (a): $t^{\prime}=0.0, \mu=0.0$, and (b) $t^{\prime}=-0.3 t, \mu=-0.9 t$. The graphs are off-set for clarity.

Thus strong interference between the impurity wavefunctions survive the filtering effect and pose new constraints on the potential scattering scenario versus more strongly correlated models[17].
In the following we show how the interference between unitary scatterers is strongly affected by the induction of a small subdominant superconducting order parameter. Thus one may utilize the quantum interference between several impurities as an alternative method to detect a small subdominant order parameter. For instance, tuning through a quantum phase transition from a $\mathrm{d}_{x^{2}-y^{2}}$ to a $\mathrm{d}_{x^{2}-y^{2}}+\mathrm{id}_{x y}$ superconductor at a critical doping level or magnetic field strength, a small $\mathrm{d}_{x y}$ order could qualitatively alter the interference pattern. For $\Delta_{x y}(\mathbf{k})=\Delta_{x y}^{0} \sin \left(k_{x}\right) \sin \left(k_{y}\right)$ with $\Delta_{x y}^{0}=2.5 \mathrm{meV}$, we compare in Fig. 4 the LDOS to the situation when $\Delta_{x y}^{0}=0$. Also we show the difference between $d+i d$ and $d+i s$ pairing symmetry for these impurity configurations $\left(\Delta_{s}^{0}=2.5 \mathrm{meV}\right)$. For most spatial configurations the secondary pairing (id or is) leads to a sharpening of the resonances but at particular positions there is a qualitative difference as shown in Fig. 4. For instance, the induction of d+id pairing (Fig. 4b) can result in three apparent resonances contrary to the ground state with pure $\mathrm{d}_{x^{2}-y^{2}}$-wave pairing (Fig. 4a). Similarly, by comparing the LDOS at $(1,1)$ (dashed lines) in Fig. 4d-f, it is evident that the interfering scatterers can provide a clear distinction between $\mathrm{d}+\mathrm{id}$ and $\mathrm{d}+\mathrm{is}$ pairing. Information of the induction of local order around the impurities can also be inferred from STM measurements of specific impurity configurations.

We turn now to the DDW state with the mean-field Hamiltonian given by[14]

$$
H=\sum_{\mathbf{k} \sigma} \xi(\mathbf{k}) c_{\mathbf{k} \sigma}^{\dagger} c_{\mathbf{k} \sigma}+i \sum_{\mathbf{k} \sigma} D(\mathbf{k}) c_{\mathbf{k} \sigma}^{\dagger} c_{\mathbf{k}+\mathbf{Q} \sigma}
$$

where $\mathbf{Q}=(\pi, \pi)$ and $D(\mathbf{k})=\frac{D_{0}}{2}\left(\cos \left(k_{x}\right)-\cos \left(k_{y}\right)\right)$. This leads to the following Greens function

$$
\hat{G}^{0}\left(\mathbf{k}, i \omega_{n}\right)=\frac{\left(\begin{array}{cc}
i \omega_{n}-\xi(\mathbf{k}+\mathbf{Q}) & -i D(\mathbf{k}) \\
i D(\mathbf{k}) & i \omega_{n}-\xi(\mathbf{k})
\end{array}\right)}{\left(i \omega_{n}-\xi(\mathbf{k})\right)\left(i \omega_{n}-\xi(\mathbf{k}+\mathbf{Q})\right)-D(\mathbf{k})^{2}}
$$

As for the DSC state we use $D_{0}=50 \mathrm{meV}$. Performing the Fourier transform $\hat{G}^{0}\left(\mathbf{r}_{i}, \mathbf{r}_{j}, i \omega_{n}\right)=$ $\sum_{\mathbf{k k}^{\prime}} \hat{G}^{0}\left(\mathbf{k}, \mathbf{k}^{\prime}, i \omega_{n}\right) e^{i \mathbf{k} \cdot \mathbf{r}_{i}-i \mathbf{k}^{\prime} \cdot \mathbf{r}_{j}}$ of the Greens function with reference to the entries of Eqn. (6) gives

$$
\begin{aligned}
\hat{G}^{0}\left(\mathbf{r}_{i}, \mathbf{r}_{j}, i \omega_{n}\right) & =\sum_{\mathbf{k}}\left[G_{11}^{0}\left(\mathbf{k}, i \omega_{n}\right)+G_{12}^{0}\left(\mathbf{k}, i \omega_{n}\right) e^{-i \mathbf{Q} \cdot \mathbf{r}_{j}}+\right. \\
G_{21}^{0}\left(\mathbf{k}, i \omega_{n}\right) e^{i \mathbf{Q} \cdot \mathbf{r}_{i}} & \left.+G_{22}^{0}\left(\mathbf{k}, i \omega_{n}\right) e^{i \mathbf{Q} \cdot\left(\mathbf{r}_{i}-\mathbf{r}_{j}\right)}\right] e^{i \mathbf{k} \cdot\left(\mathbf{r}_{i}-\mathbf{r}_{j}\right)}
\end{aligned}
$$


summed over the reduced Brillouin zone. In the DDW phase the single impurity resonance condition, $1=$ $U \operatorname{Re}\left[G^{0}\left(0, i \omega_{n}\right)\right]$, has been previously studied without the filtering effect[15]. In Fig. 5a-b we show the LDOS in the DDW state for the two different sets of band parameters including the filter. In the nested case (Fig. 5a) the expected single resonance is clearly seen. The tunnelling filter shifts the LDOS maximum from the nearest neighbors to the impurity site and induce a second maximum on the nextnearest neighbors. This weight redistribution is identical to the situation in the DSC phase[8]. Thus when comparing Fig. 1a and $5 \mathrm{a}$ it is clear that a single nonmagentic impurity cannot easily distinguish the DSC and DDW phases. Another difference between the DSC and DDW states is the position of the resonance in the unitary limit, $U \rightarrow \infty$.
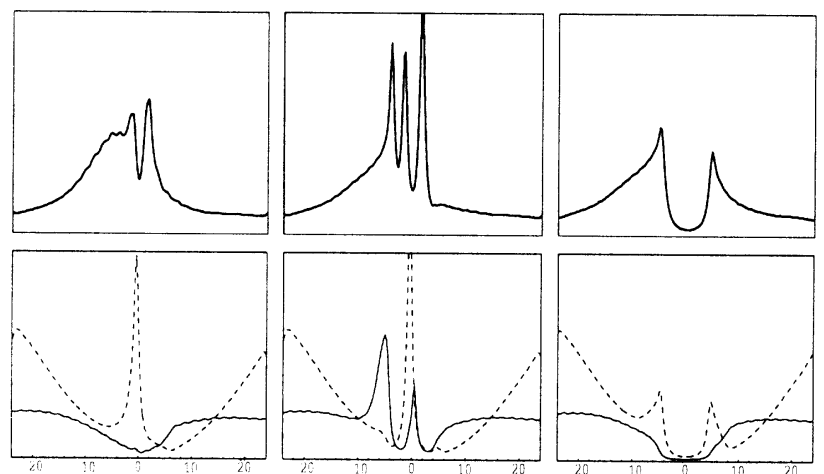

Figure 4. Top row: DOS at $(0,0)$ for two nonmagnetic impurities at $(0,0)$ and $(2,4)$. Bottom row: DOS at $(0,0)$ (solid line) and $(1,1)$ (dashed line) for three nonmagnetic impurities at $(-1,1),(1,-$ 1) and $(-1,-1)$. Pairing symmetry: (a) and (d) $\mathrm{d}_{x^{2}-y^{2}}$, (b) and (e) $\mathrm{d}_{x^{2}-y^{2}}+\mathrm{id}_{x y}$, (c) and (f) $\mathrm{d}_{x^{2}-y^{2}}+\mathrm{is}$.

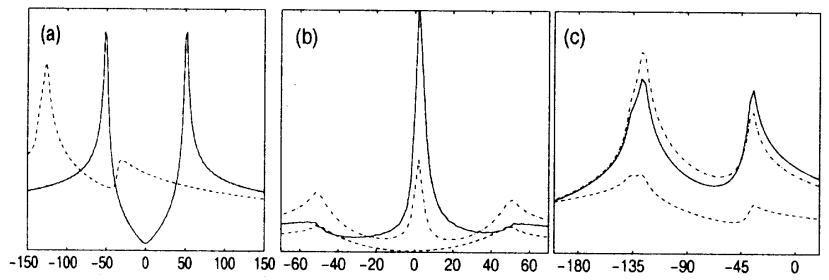

Figure 5.(a-b) same as Fig. 1 (DDW). (c) DOS at $(0,0)$, impurities at $(0,0),(2,0)$ for the DDW (solid) or DSC (dashed).

For the DDW phase the resonance energy approaches minus the chemical potential, $\omega=-\mu$, whereas it approaches the Fermi level in the DSC phase[15]. The different resonance energy (as $U \rightarrow \infty$ ) arises from the way the chemical potential enters the bands of the clean DDW $\left(E_{ \pm}(\mathbf{k})=\left|\sqrt{\epsilon(\mathbf{k})^{2}+D(\mathbf{k})^{2}} \pm \mu\right|\right)$ and DSC $\left(E_{ \pm}(\mathbf{k})=\right.$ $\left.\sqrt{(|\epsilon(\mathbf{k})| \pm \mu)^{2}+\Delta(\mathbf{k})^{2}}\right)$ states[15]. This also results in a large overlap between the impurity resonance and the continuum when $\mu \neq 0$ in the DDW state. Thus the peaks in Fig. $5 b$ are not impurity resonances but the shifted gap edges (note energy range). The impurity can however slightly modify the amplitude of these peaks. This is also evident from the two impurity case shown in Fig. 6b. As expected the quantum interference is very weak but clearly distinct from the equivalent impurity configurations in Fig. 3. This is contrary to the nested case shown in Fig. 6a. Here there is strong quantum interference between the two nonmagnetic impurities. However, by comparison with Fig. 3a it is clear that the additional resonance states in the DSC again allows one to distinguish this phase from the DDW order.
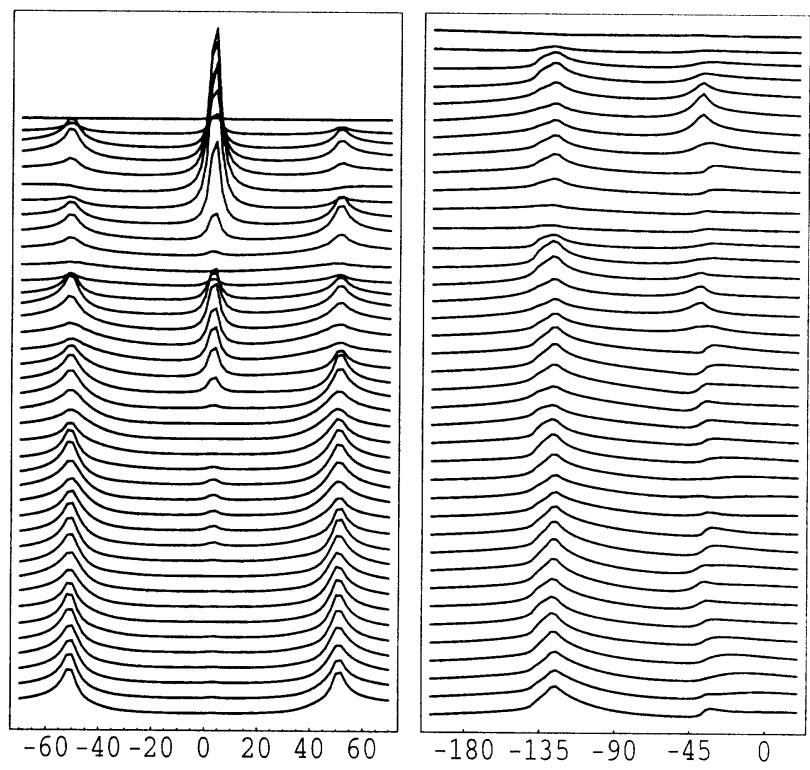

Figure 6. Same as Fig. 3 but for the d-density wave state.

Fig. 5e shows other impurity and STM tip positions where this difference is particularly striking.

In summary we propose that a systematic study around two nonmagnetic impurities can clearly distinguish the DSC and DDW phases. The impurities are modelled as potential scatterers and the results pose further tests on this approach. It would also be interesting to study similar multiple impurity interference effects within other pseudo-gap models and within other proposed scenarios for the resonances around nonmagnetic impurities in d-wave superconductors.

This work is supported by the Danish Technical Research Council via the Framework Prog. on Superconductivity.

\section{References}

[1] L. Yu, Acta Phys. Sin. 21, 75 (1965); H. Shiba, Prog. Theo. Phys. 40, 435 (1968).

[2] A.V. Balatsky, M.I. Salkola, and A. Rosengren, Phys. Rev. B 5115547 (1995); M.I. Salkola, A.V. Balatsky, and D.J. Scalapino, Phys. Rev. Lett. 77, 1841 (1996).

[3] A. Yazdani et al., Phys. Rev. Lett. 83, 176 (1999). 
[4] S.H. Pan et al., Nature 403, 746 (2000).

[5] E.W. Hudson et al., Nature 411, 920 (2001).

[6] J.E. Hoffman et al., Science 297, 1148 (2002); K. McElroy et al., Nature 422, 592 (2003)

[7] Q.-H. Wang and D.-H. Lee, Phys. Rev. B 67, 020511 (2003).

[8] I. Martin, A.V. Balatsky, and J. Zaanen, Phys. Rev. Lett. 88, 097003 (2002)

[9] H. Alloul et al., Phys. Rev. Lett. 67, 3140 (1991); J. Bobroff et al., Phys. Rev. Lett. 83, 4381 (1999); A.V. Mahajan et al., Europhys. Lett. 46, 678 (2000).

[10] H.C. Manoharan, C.P. Lutz, and D.M. Eigler, Nature 403, $512(2000)$

[11] D. Morr, and N.A. Stavropoulos, Phys. Rev. B 66, 140508 (2002).

[12] L. Zhu, W.A. Atkinson, and P.J. Hirschfeld, Phys. Rev. B 67, 094508 (2003).
[13] B.M. Andersen, and P. Hedegård, Phys. Rev. B 67172505 (2003).

[14] S. Chakravarty et al., Phys. Rev. B 63, 094503 (2001).

[15] J-X. Zhu et al., Phys. Rev. Lett. 87, 197001 (2001); Q-H. Wang, Phys. Rev. Lett. 88, 057002 (2002); D. Morr, Phys. Rev. Lett. 89, 106401 (2002).

[16] H.K. Nguyen, and S. Chakravarty, Phys. Rev. B 65, 180519 (2002); S. Chakravarty, H.-Y. Kee, and C. Nayak, Int. J. Mod. Phys. B 15, 2901 (2001).

[17] Z. Wang, and P.A. Lee, Phys. Rev. Lett. 89, 217002 (2002); A. Polkovnikov, S. Sachdev, and M. Vojta, Phys. Rev. Lett. 86, 296 (2001); K. Park, cond-mat/0203142.

[18] T. Chen et al., Phys. Rev. Lett. 84, 147 (2000); C. Howald et al., Phys. Rev. B 64, 100504 (2001); K.M. Lang et al., Nature 415, 412 (2002)

[19] B.M. Andersen, P. Hedegård, and H. Bruus, Phys. Rev. B 67, 134528 (2003); J. Low. Temp. Phys. 131, 281 (2003). 